\title{
The Association of Insomnia with Depression and Anxiety Symptoms in Patients Undergoing Noncardiac Surgery
}

This article was published in the following Dove Press journal: Neuropsychiatric Disease and Treatment

\section{Xiao Huang \\ Dan Wu \\ An-Shi Wu \\ Chang-Wei Wei \\ Jian-Dong Gao (D)}

Department of Anesthesia, Beijing ChaoYang Hospital, Capital Medical University, Beijing, 100020, People's Republic of China
Correspondence: Jian-Dong Gao; Chang-Wei Wei

Department of Anesthesia, Beijing ChaoYang Hospital, Capital Medical University, No. 8 South Worker's Stadium Road, Chao-Yang District, Beijing, 100020,

People's Republic of China

Tel +86 1085231330

Fax $+86108523 \mid 463$

Email gaojiandong66_dr@163.com; weichangwei53fg@163.com
Objective: Insomnia is common in patients undergoing surgery. It can increase the rate of postoperative complications, interfere with patient recovery, and decrease hospital satisfaction. However, there are few studies on perioperative insomnia. This study was conducted to investigate the differences in the demographic, health status, and clinical characteristics of patients with and without insomnia postoperatively, and to identify the potential risk factors of insomnia.

Methods: There were 299 non-cardiac surgery patients, 165 females, and 134 males, with a mean age of 55 years, enrolled in the study. The Insomnia Severity Index (ISI), Patient Health Questionnaire-9 (PHQ-9), Generalized Anxiety Disorder 7 (GAD-7), and Montreal Cognitive Assessment (MoCA) were administered to all the patients preoperatively. The Visual Analogue Scale (VAS) was used preoperatively, and at the end of the surgery, and then one day, two days, and three days after surgery. The PHQ-9, the GAD-7, and the ISI were repeated three days after surgery. Insomnia was diagnosed by the ISI as being a score of 8-28 (mild: 8-14; moderatesevere: 15-21; severe: 22-28). The patients were divided into group A (with insomnia, $\mathrm{N}=78$ ) and group $\mathrm{B}$ (without insomnia, $\mathrm{N}=221$ ) according to their ISI score three days after surgery. The general clinical data of the two groups were analyzed first, and then binary logistic regression analysis was conducted to assess the risk factors of insomnia.

Results: A total of 299 non-cardiac surgery patients with a mean age of 55 years were enrolled in the study. Of the included patients, the number of females was 165 and the number of the male was 134 . The incidence of insomnia at 3 days postoperatively was $26.1 \%$ (78/299). The average points that group A patients scored in the ISI, PHQ-9, and the GAD-7 were significantly higher than those in group B. The VAS score three days after surgery was significantly higher in group A. The PHQ-9 and the GAD-7 three days after surgery showed significantly higher depression and anxiety scores in group A. Logistic regression showed that the ISI $(p<0.001,95 \% \quad \mathrm{CI}=1.218-1.500)$ and the GAD-7 $(p=0.003,95 \%$ $\mathrm{CI}=1.041-1.218)$ preoperatively, and the PHQ-9 postoperatively $(p<0.001,95 \%$ $\mathrm{CI}=1.226-1.555)$, were risk factors of insomnia.

Conclusion: Insomnia is common and can worsen after surgery. The present study suggests that depression and anxiety are risk factors for insomnia after surgery. There is a need for further research and the development of strategies for depression and anxiety management to ensure better sleep quality for patients, which will be of significant benefit to their health.

Clinical Trial Registration: The study was registered at clinical trial (NCT04027751); Trial registration: clinical trial, NCT04027751. Registered 22 July 2019; https://clinicaltrials. gov/ct2/show/NCT04027751?cond=NCT04027751\&cntry=CN\&draw=2\&rank=1.

Keywords: insomnia, depression, anxiety, postoperative management, undergoing surgery, noncardiac surgery 


\section{Introduction}

Sleep problems are serious, damaging comorbidities. ${ }^{1}$ They can influence mood and emotional or behavioral states and are complicated in psychopathology. Significantly strong partial associations of sleep disturbance with anxiety, depression, and insomnia, and a modest partial association with sleepiness have been detected. ${ }^{2}$ Insomnia is one of the most common sleep disorders and a known risk factor for the incidence and severity of depression, recurrence of depressive episodes, and even suicide. ${ }^{3}$ It often coexists with depression and anxiety, and there is an intricate relationship between them in nonsurgical patients. Insomnia is common in patients during the perioperative period, and for postoperative recovery, it has negative consequences, ${ }^{4}$ such as pain and fatigue, which reduce the quality of life, ${ }^{5}$ especially in those with tumor surgery. ${ }^{6}$

Although insomnia is one of the most prevalent health problems, it is often neglected, and clinicians may be reluctant to address insomnia because it has many potential causes and they may be unfamiliar with behavioral treatments. Little is known about the sleep condition during the perioperative period, and few studies have focused on possible associations between sleep disorders and clinical characteristics in patients undergoing surgery. There is a need to screen for sleep problems and address sleep disturbances routinely in clinical practice. Further studies are warranted concerning factors that contribute to sleep disturbances and how they can best be treated. Due to the potential impact of insomnia and its strong connection with depression in non-surgical patients, the main aim of the present study was to identify the potential risk factors of insomnia and explore the association of insomnia with depression and anxiety in patients undergoing noncardiac surgery.

\section{Method}

\section{Participants}

The study was a retrospective cohort study (clinical trial registration number: NCT04027751). We carefully selected participants from the Beijing Chaoyang Hospital, Capital Medical University. Recruitment started in September 2019 and was completed in March 2020. The study was approved by the Regional Ethical Review Committee at the hospital and complied with the Declaration of Helsinki (2019-ke-273).
Participants were recruited according to the following inclusion criteria: between 18 to 85 years of age; able to read, write and understand; willing and able to complete pre-and postoperative surveys; had undergone surgery in Beijing Chaoyang Hospital. The exclusion criteria were as follows: diagnosed with a sleep disorder previously and had a history of medication for it; using antidepressant or antipsychotic drugs; having used sedatives preoperatively; undergoing ambulatory, non-general anesthesia surgery or cancellation of surgery; a history of substance abuse; a history of prescription or supplemental sleeping aids; suffering from clinical conditions (such as mucositis, severe pain, nausea, dyspnea, and vomiting) or emotional states (crying, apathy, aggression), which would prevent them from taking part in an interview; did not consent to fill out a questionnaire. It was established whether these criteria were met during the interview at the time of the data collection.

The researcher asked the potential participants if they felt comfortable answering questions at that time and informed them that they were free to decline to participate. All patients signed a written consent to participate in this research. The participants were interviewed one day before the operation, at the end of the operation, two days after the operation, and three days after the operation. A sample of 299 patients was finally enrolled in the study. The patients were divided into two groups according to whether or not they had insomnia postoperatively, group A (insomnia, $\mathrm{N}=78$ ) and group $\mathrm{B}$ (without insomnia, $\mathrm{N}=221$ ). This study aimed to explore research questions regarding the sleep condition preoperatively, depression, anxiety, and cognition, as well as the surgical prognosis. A flow chart of the study is shown in Figure 1.

\section{Tools}

The presence of sleep disturbances was evaluated by using the Insomnia Severity Index (ISI). The ISI is a tool for measuring sleep impairment, which permits unrestricted use and distribution. The items are scored from 0 to 28 , with higher scores indicating worse sleep quality. The ISI contains seven items on sleep problems including trouble falling asleep, waking up during the night, waking up earlier than planned, trouble getting back to sleep, and sleep quality for the past month (on a scale of $0-4$ ). ISI is a valid screening instrument for detecting insomnia among patients, ${ }^{7}$ and we defined insomnia as being a score of 8-28 (mild: 8-14; moderate-severe: 15-21; severe: 22-28). 


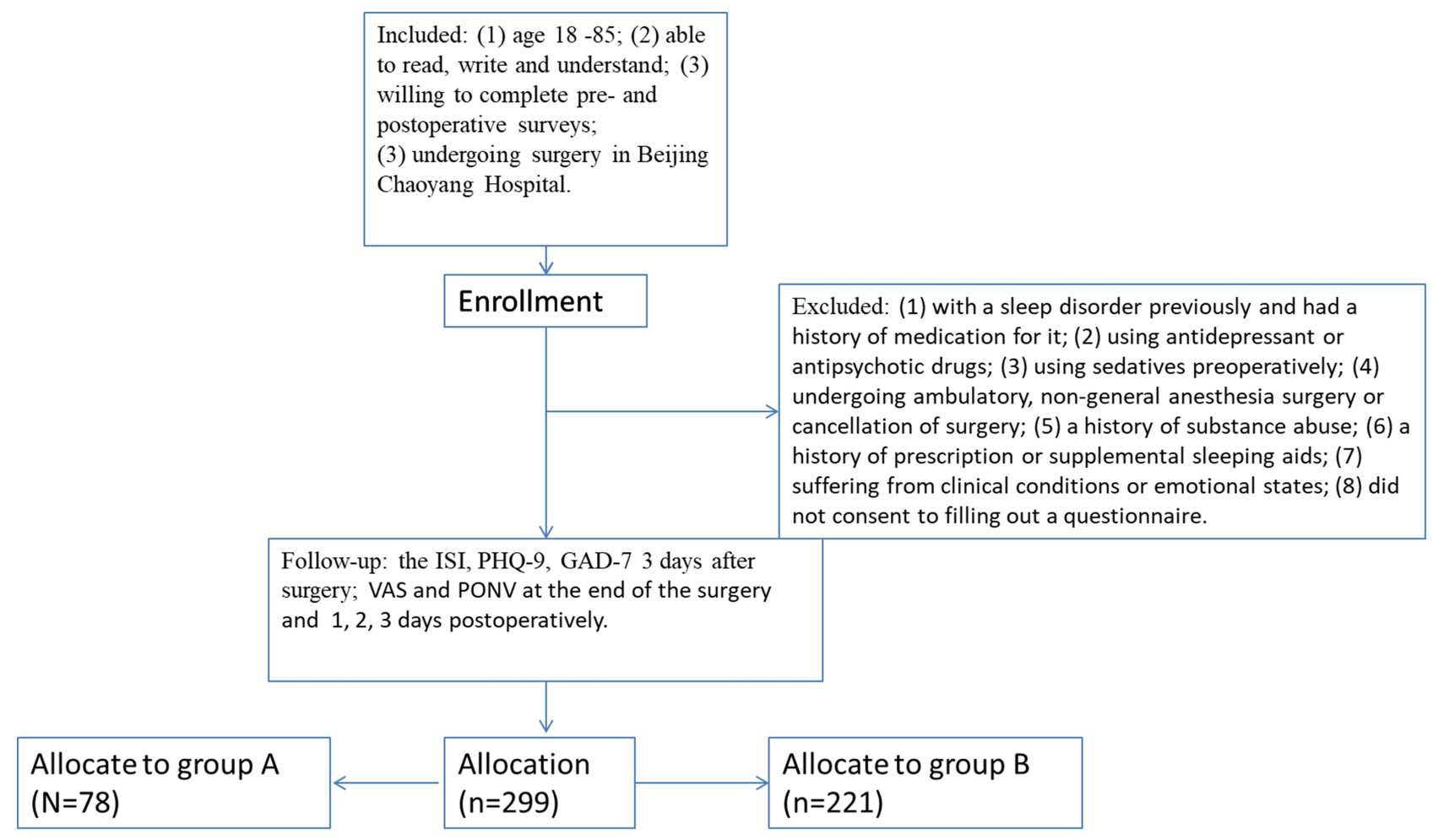

Figure I The flow chart of the study.

Abbreviations: ISI, Insomnia Severity Index; PHQ-9, Patient Health Questionnaire-9; GAD-7, Generalized Anxiety Disorder-7; VAS, Visual Analog Scale.

The Patient Health Questionnaire-9 (PHQ-9) is a tool for screening and diagnosing the degree of depression. It consists of nine items and is used to measure how people view their lives and how they use their resources of resistance to maintain and improve their health. Each item of the PHQ-9 is rated on a 4-point scale, giving a total score ranging from 0 to 27 . Higher scores indicate increased severity of symptoms and an increased likelihood of major depressive disorders. The validity and utility of PHQ-9 in the diagnosis of depression and its efficiency of use are generally recognized. ${ }^{8,9}$

Generalized Anxiety Disorder 7 (GAD-7) is a widely used measure of the characteristics of anxiety disorders, and it is being increasingly used in research and clinical practice. ${ }^{10}$ Patients rate the frequency of their symptoms within the previous two weeks on a four-point scale ranging from "not at all" to "almost every day". The GAD-7 consists of seven items and the score range from 0 to 21 with higher scores indicating higher GAD symptomatology. The GAD-7 scale is a self-administered tool used to screen, rate, and monitor the outcome of anxiety disorders in primary healthcare setups and is considered to be a rapid yet valid way of diagnosing GAD with accuracy. ${ }^{11}$
We used the Montreal Cognitive Assessment (MoCA) to screen for cognitive disorders. It includes the assessment of attention, concentration, executive function, memory, and language. The MoCA consists of 11 items, in total, and scores range from 0 to 30 . The MoCA is said to be more sensitive than the Mini-Mental State Examination in detecting mild cognitive impairment, and to test executive function more extensively. ${ }^{12}$

The Visual Analogue Scale (VAS) is used to evaluate the severity of pain in patients, with 0 being "no pain at all" and 10 being "my pain is as bad as it could be". The VAS has been proved to be an effective and reliable method of evaluating acute and chronic pain, especially in elderly patients.

The PHQ-9, the GAD-7, the ISI, and the MoCA were assessed at the baseline. The VAS was assessed preoperatively, at the end of the surgery, one day after surgery, two days after surgery, and three days after surgery. The PHQ-9, the GAD-7, and the ISI were reassessed three days after surgery.

\section{Data Collection}

Patients provided information on sociodemographic variables at enrolment. Demographic data were collected for 
gender, age, years of education, body mass index (BMI), history of diabetes, hypertension, smoking and drinking habits, type of surgery, American Society of Anesthesiologists (ASA) classification, operation time, intraoperative urine volume, blood loss, the use of the patient-controlled analgesia, the incidence of postoperative nausea and vomiting (PONV), and the length of hospital stay, in addition to the data from the scales above.

\section{Statistical Analysis}

Data management and statistical analyses were performed using SPSS Statistics software version 25 (IBM Corp., Armonk, New York, United States). The data were presented as means \pm standard error (SEM) for continuous variables and an Independent-Samples $t$-Test was used if they were normally distributed, but if not, they were presented as median [interquartile range], and a MannWhitney U-test was used. The data were shown as percentages (\%) for categorical variables and a Chi-Square test was used. We also used an ISI scale score cut-off of seven for expressing sleep impairment in a clinically applicable way and for quantifying its risks associated with health status and disease severity. The observed distribution of insomnia was summarized in defined groups based on the scores from the ISI as follows: none (0-7), mild (8-14), moderate (15-21), and severe (22-28). ${ }^{13}$ Depression was rated in PHQ-9 at five levels: none (0-4), mild (5-9), moderate (10-14), moderate-severe (15-19, and severe (20-27). Anxiety was graded according to the score of the GAD-7: none (0-4), mild (5-9), moderate (10-13), moderate-severe (14-18), and severe (19-21). Finally, cognition impairment was rated by the MoCA: none (26-$30)$, mild (18-25), and moderate $(\leq 17) .{ }^{14,15}$

In the present study, patients were divided into two groups according to their ISI. The differences in clinical data between the two groups were analyzed first. All variables that showed a statistically significant association with insomnia in univariate analysis were included in the multivariate logistic regression. Postoperative insomnia was the dependent variable in the regression, and the preoperative sleep, anxiety, depression, and postoperative pain, anxiety, and depression were independent variables. A binary logistic regression analysis was conducted to assess which factors were significantly associated with insomnia. Receiver operator characteristic (ROC) curves were created by plotting sensitivity against 1 -specificity and assessing the total area under the curve for each scale. A $p$-value less than 0.05 on two sides was adopted as the critical level for all the tests. All the participants provided written informed consent, and ethical approval was provided by the local medical ethics committee of the Beijing Chaoyang Hospital, Capital Medical University.

\section{Results}

A total of 299 patients (female $=165$, male $=134$ ) with a mean age of 55 years were evaluated in this study. The demographic data of all the participants are shown in Table 1. There were no differences concerning demographic

Table I Preoperative Patient Characteristics by Status of ISI 3 Days After Surgery

\begin{tabular}{|c|c|c|c|c|}
\hline Variables & All $(\mathbf{N}=299)$ & Group $A(\mathbf{N}=78)$ & Group B $(N=221)$ & $P$-value \\
\hline Sex-female; n (\%) & $165(55.2)$ & $44(56.4)$ & $|2|(54.8)$ & 0.800 \\
\hline Age, years & $55.2 \pm 0.8$ & $56.9 \pm 1.5$ & $54.6 \pm 1.0$ & 0.224 \\
\hline Years of education, years & $11.3 \pm 0.2$ & $1 \mathrm{I} .0 \pm 0.4$ & $11.3 \pm 0.2$ & 0.556 \\
\hline Body mass index, $\mathrm{kg} / \mathrm{m}^{2}$ & $24.6 \pm 0.2$ & $24.0 \pm 0.4$ & $24.8 \pm 0.2$ & 0.126 \\
\hline VAS before surgery & $0(0,0)$ & $0(0,2.0)$ & $0(0,0)$ & 0.061 \\
\hline Diabetes & $52(17.4)$ & $13(16.7)$ & $39(17.6)$ & 0.844 \\
\hline Hypertension & $105(35.1)$ & $27(34.6)$ & $78(35.3)$ & 0.914 \\
\hline Current smoking; n (\%) & $47(15.7)$ & $7(9.0)$ & $40(18.1)$ & 0.137 \\
\hline Alcohol use; n (\%) & $19(6.4)$ & $4(5.1)$ & $15(6.8)$ & 0.712 \\
\hline ISI of baseline & $3.9 \pm 0.2$ & $7.5 \pm 0.6$ & $2.7 \pm 0.2$ & $<0.001$ \\
\hline PHQ-9 of baseline & $2.6 \pm 0.3$ & $4.8 \pm 1.1$ & $1.8 \pm 0.2$ & 0.008 \\
\hline GAD-7 of baseline & $2.1 \pm 0.2$ & $3.0 \pm 0.4$ & $1.7 \pm 0.2$ & 0.005 \\
\hline $\mathrm{MoCA}$ & $25.3 \pm 0.2$ & $24.8 \pm 0.4$ & $25.5 \pm 0.2$ & 0.117 \\
\hline
\end{tabular}

Notes: The data were presented as means \pm standard error, SEM for continuous variables, and $\mathrm{t}$ value are presented; as percentages (\%) for categorical variables and $\chi$ value are presented; as median [interquartile range] and Mann-Whitney U-test are presented.

Abbreviations: VAS, Visual Analog Scale; ISI, Insomnia Severity Index; PHQ-9, Patient Health Questionnaire-9; GAD-7, Generalized Anxiety Disorder; MoCA, Montreal Cognitive Assessment. 
Table 2 Baseline Classification of Scales

\begin{tabular}{|c|c|c|c|c|}
\hline Variables & Patients (N=299) & Group A & Group B & $P$-value \\
\hline ISI; n (\%) (N=299) & & & & $<0.001$ \\
\hline Non & $243(81.3)$ & $39(50)$ & $204(92.3)$ & \\
\hline Mild & $46(15.4)$ & $29(37.2)$ & $17(7.7)$ & \\
\hline Moderate & $10(3.3)$ & $10(12.8)$ & $0(0)$ & \\
\hline PHQ-9; n (\%) (N=298) & & & & $<0.001$ \\
\hline Non & $244(81.9)$ & $49(62.8)$ & $195(88.6)$ & \\
\hline Mild & $38(12.8)$ & $21(26.9)$ & $17(7.7)$ & \\
\hline Moderate & $16(5.4)$ & $8(10.3)$ & $8(3.6)$ & \\
\hline GAD-7; n (\%) (N=299) & & & & 0.022 \\
\hline Non & $244(8 I .6)$ & $56(72.8)$ & $188(85.1)$ & \\
\hline Mild & $44(14.7)$ & $17(2 \mid .8)$ & $27(12.2)$ & \\
\hline Moderate & $9(3.0)$ & $5(6.4)$ & $4(1.8)$ & \\
\hline Moderate-severe & $2(0.7)$ & $0(0)$ & $2(0.9)$ & \\
\hline MoCA; n (\%) $(\mathrm{N}=296)$ & & & & 0.336 \\
\hline Non & $162(54.7)$ & $37(48.7)$ & $125(56.8)$ & \\
\hline Mild & $124(41.9)$ & $35(46.1)$ & $89(40.5)$ & \\
\hline Moderate & $10(3.4)$ & $4(5.3)$ & $6(2.7)$ & \\
\hline
\end{tabular}

Note: Percentages (\%) for categorical variables and $\chi$ value are presented.

Abbreviations: ISI, Insomnia Severity Index; PHQ-9, Patient Health Questionnaire-9; GAD-7, Generalized Anxiety Disorder-7; MoCA, Montreal Cognitive Assessment.

characteristics (all $p>0.05$ ), but the average points group A patients scored in the ISI, PHQ-9, and the GAD-7 were significantly higher than those in group $\mathrm{B}(p<0.05)$. Baseline classifications of insomnia, depression, anxiety, and cognitive impairment are shown in Table 2. There were 46 patients diagnosed with mild insomnia, 29 (37.3\%) of them being in group A and $17(7.7 \%)$ in group B, and 10 $(12.8 \%)$ patients were diagnosed with moderate insomnia $(p<0.05)$. The number of patients diagnosed with depression and anxiety in group A was also significantly higher than that in group B $(p<0.05)$ (see Table 2).

In Table 3 , it can be seen that there were no significant differences in intraoperative clinical information between patients with and without insomnia.; no significant difference was found in the type of surgery, ASA classification, intraoperative urine volume, blood loss, operation time, or the VAS scores at the end of the surgery (all $p>0.05$ ).

Table 4 shows the postoperative variables. The VAS scores three days after surgery were significantly higher in group A $(p<0.05)$, but no significant difference between the two groups was found in the VAS scores at any other time points. When comparing patients with insomnia to the controls, the PHQ-9 and the GAD-7 three days after surgery showed significantly higher depression and anxiety scores in group A $(p<0.05)$. The two groups were matched for postoperative analgesia, the incidence of postoperative nausea and vomiting, and the length of hospital stay.

We used logistic regression to adjust for the scale scores, namely, the ISI of the baseline, the VAS scores three days after surgery, and the pre-and postoperative PHQ-9 and GAD-7. A significant difference was also found in the ISI $(p<0.001,95 \% \mathrm{CI}=1.218-1.500)$, the GAD-7 $(p=0.003,95 \%$ $\mathrm{CI}=1.041-1.218)$ preoperatively, and the PHQ-9 $(p<0.001$, 95\% CI=1.226-1.555) postoperatively (see Table 5). An analysis of the area under the curve for the ROC of the tests demonstrated that a PHQ-9 three days after surgery was best for detecting insomnia (area under the curve $[\mathrm{AUC}]=0.818$ ) (see Figure 2).

\section{Discussion}

This study was undertaken to observe the perioperative clinical data of postoperative insomniacs and to examine predictors of insomnia in patients after surgery and the relationships between sleep, depression, and anxiety. Sleep quality was quantified using the validated ISI instrument in the present study. The patients in our study exhibited insomnia, with $18.7 \%$ of the patients before surgery and $26.1 \%$ of the patients after surgery suffering from insomnia. We can see even poorer sleep postoperatively. We also found that anxiety preoperatively and depression postoperatively were risk factors of insomnia. 
Table 3 Intraoperative Variables by Status of ISI 3 Days After Surgery

\begin{tabular}{|c|c|c|c|c|}
\hline Variables & All (N=299) & Group A $(\mathbf{N}=78)$ & Group $B(N=221)$ & $P$-value \\
\hline $\begin{array}{l}\text { Type of surgery; } \mathrm{n}(\%) \\
\text { Thoracic surgery } \\
\text { Orthopaedic surgery } \\
\text { urology } \\
\text { Gynecologic surgery } \\
\text { Gastrointestinal surgery } \\
\text { Others }\end{array}$ & $\begin{array}{c}67(22.4) \\
45(15.1) \\
71(23.7) \\
45(15.1) \\
28(9.4) \\
43(14.4)\end{array}$ & $\begin{array}{c}25(32.1) \\
14(17.9) \\
15(19.2) \\
5(6.4) \\
8(10.3) \\
11(14.1)\end{array}$ & $\begin{array}{c}42(19.0) \\
31(14.0) \\
56(25.3) \\
40(18.1) \\
20(9.0) \\
32(14.5)\end{array}$ & 0.117 \\
\hline $\begin{array}{l}\text { ASA classification; } \mathrm{n}(\%) \\
\text { Class I } \\
\text { Class II } \\
\text { Class III } \\
\text { Intraoperative urine volume, } \mathrm{mL} \\
\text { Intraoperative blood loss, } \mathrm{mL} \\
\text { Operation time, min } \\
\text { VAS at the end of surgery }\end{array}$ & $\begin{array}{c}36(12.0) \\
190(63.5) \\
73(24.4) \\
426.8 \pm 22.2 \\
110.9 \pm 8.1 \\
160.3 \pm 4.6 \\
2.0(1.0,3.0)\end{array}$ & $\begin{array}{c}7(9.0) \\
50(64.1) \\
21(26.9) \\
465.0 \pm 50.9 \\
104.4 \pm 14.3 \\
159.2 \pm 9.3 \\
2.0(1.0,4.0)\end{array}$ & $\begin{array}{c}29(13.1) \\
140(63.3) \\
52(23.5) \\
412.4 \pm 23.8 \\
113.4 \pm 9.8 \\
160.7 \pm 5.3 \\
2.0(1.0,3.0)\end{array}$ & $\begin{array}{l}0.291 \\
0.622 \\
0.892 \\
0.289\end{array}$ \\
\hline
\end{tabular}

Abbreviations: ASA, American Society of Anesthesiologists; VAS, Visual Analog Scale.

Research has shown that insomnia is highly prevalent and associated with an unhealthy physical and mental state. ${ }^{16}$ Our study provides support to the claim that patients suffering from anxiety and depression are more likely to experience insomnia after surgery. Anxiety and depression have often been shown to be the primary risk factors of insomnia. Sleep disturbance is also commonplace in patients, and its association with psychological

Table 4 Postoperative Variables by Status of ISI 3 Days After Surgery

\begin{tabular}{|c|c|c|c|c|}
\hline Variables & All $(\mathbf{N}=299)$ & Group $A(\mathbf{N}=78)$ & Group $B(N=22 I)$ & P-value \\
\hline Postoperative analgesia; n (\%) & & & & 0.100 \\
\hline Non & $93(3 \mathrm{I} .1)$ & $22(39.1)$ & 71 (42.6) & \\
\hline $\mathrm{PCIA}$ & $179(59.9)$ & $44(55.1)$ & $135(49.4)$ & \\
\hline PCEA & $27(9.0)$ & $12(5.8)$ & $15(8.0)$ & \\
\hline VAS I day after surgery & $2.0(1.0,4.0)$ & $3.0(1.0,4.5)$ & $2.0(1.0,4.0)$ & 0.101 \\
\hline VAS 2 days after surgery & $2.0(0,3.0)$ & $2.0(0,3.3)$ & $2.0(0,3.0)$ & 0.904 \\
\hline VAS 3 days after surgery & $1.0(0,3.0)$ & $2.0(0,3.0)$ & $1.0(0,2.0)$ & 0.014 \\
\hline PONV Iday after surgery; $n(\%)(\mathrm{N}=294)$ & & & & 0.722 \\
\hline 0 & $258(87.8)$ & $69(88.4)$ & $189(87.4)$ & \\
\hline I & $9(3.1)$ & $3(4.3)$ & $6(4.1)$ & \\
\hline 2 & $3(1.0)$ & $0(0)$ & $3(0.9)$ & \\
\hline 3 & $24(8.2)$ & $6(7.2)$ & $18(7.6)$ & \\
\hline PONV 2 days after surgery; $n(\%)(N=294)$ & & & & 0.506 \\
\hline 0 & $282(95.9)$ & $76(90.6)$ & $206(97.0)$ & \\
\hline I & $6(2.0)$ & $2(6.3)$ & $4(1.5)$ & \\
\hline 2 & $\mathrm{I}(0.3)$ & $0(0)$ & $I(0)$ & \\
\hline 3 & $5(1.7)$ & $0(0)$ & $5(1.5)$ & \\
\hline ISI 3 days after surgery & $5.0 \pm 0.3$ & $11.6 \pm 0.4$ & $2.7 \pm 0.2$ & $<0.001$ \\
\hline PHQ-9 3 days after surgery & $2.7 \pm 0.2$ & $5.7 \pm 0.5$ & $1.7 \pm 0.2$ & $<0.001$ \\
\hline GAD-7 3 days after surgery & $1.7 \pm 0.2$ & $3.2 \pm 0.6$ & $1.2 \pm 0.2$ & 0.001 \\
\hline Length of hospital stay, days & $1 \mathrm{I} .4 \pm 0.4$ & $11.3 \pm 0.8$ & $11.5 \pm 0.5$ & 0.903 \\
\hline
\end{tabular}

Abbreviations: PCIA, patient-controlled intravenous analgesia; PCEA, patient-controlled epidural analgesia; VAS, Visual Analog Scale; PONV, postoperative nausea and vomiting; PONV, 0 indicate no nausea or vomiting; I, indicate nausea; 2, indicate vomiting; 3, indicate both nausea and vomiting; ISI, Insomnia Severity Index; PHQ-9, Patient Health Questionnaire-9; GAD-7, Generalized Anxiety. 
Table 5 Summary of Logistic Regression with ISI 3 Days After Surgery as Dependent Variable

\begin{tabular}{|l|c|c|c|}
\hline Variables & OR & $\mathbf{9 5 \% C l}$ & $\boldsymbol{P}$ \\
\hline ISI of baseline & 1.352 & $1.218-1.500$ & $<0.001$ \\
PHQ-9 of baseline & 1.023 & $0.959-1.091$ & 0.491 \\
GAD-7 of baseline & 1.126 & $1.041-1.218$ & 0.003 \\
VAS 3 days after surgery & 1.034 & $0.866-1.234$ & 0.715 \\
PHQ-9 3 days after surgery & 1.381 & $1.226-1.555$ & $<0.001$ \\
GAD-7 3 days after surgery & 0.953 & $0.854-1.064$ & 0.391 \\
\hline
\end{tabular}

Abbreviations: ISI, Insomnia Severity Index; PHQ-9, Patient Health Questionnaire-9; GAD-7, Generalized Anxiety Disorder-7; VAS, Visual Analog Scale.

problems has negative implications, such as the potential disruption to cardiac rehabilitation efforts. ${ }^{17} \mathrm{~A}$ metaanalysis indicated that short and long sleep duration is significantly associated with an increased risk of depression in adults. ${ }^{18}$ Depression and anxiety in elderly Asian patients are associated with several sleep disorders with a somewhat different profile. ${ }^{19}$ Although the relationship between insomnia and depression has been studied extensively, $^{20}$ little is known about the association between sleep quality and depression, or between insomnia and anxiety, in surgical patients.

Similar to our findings, one study showed that depression and symptoms related to menopause were independent predictors for poor sleep quality. ${ }^{21}$ Overall, patients with sleep disturbance were more likely to develop anxiety compared to those without a history of sleep disturbance. Thus, insomnia may be a key pathology in anxiety-related disorders. However, we are unable to assign causal directionality to the association between sleep and disease characteristics in these patients. Although poor sleep and depression and anxiety seem to be strongly related, it is not clear if poor sleep causes depression and anxiety or vice versa. Moreover, the biological processes underlying this causal relationship are not yet fully understood. Patients with insomnia have fragmented REM sleep, which probably interferes with the basal processes of emotion regulation, and this interaction could lead to depression. Although preliminary results seem to support this hypothesis, there are still not enough data to draw valid conclusions. Thus, to address this issue, more studies are needed in a longitudinally-followed population sample of patients.

Contrary to our findings, $\mathrm{Ju}$ et $\mathrm{al},{ }^{22}$ reported that sleep duration and sleep quality may play a role in cognitive performance in healthy older adults. Other findings support a role for sleep disturbances in the development of cognitive impairment ${ }^{23,24}$ and suggest sleep plays a crucial part in brain aging. However, we did not find any

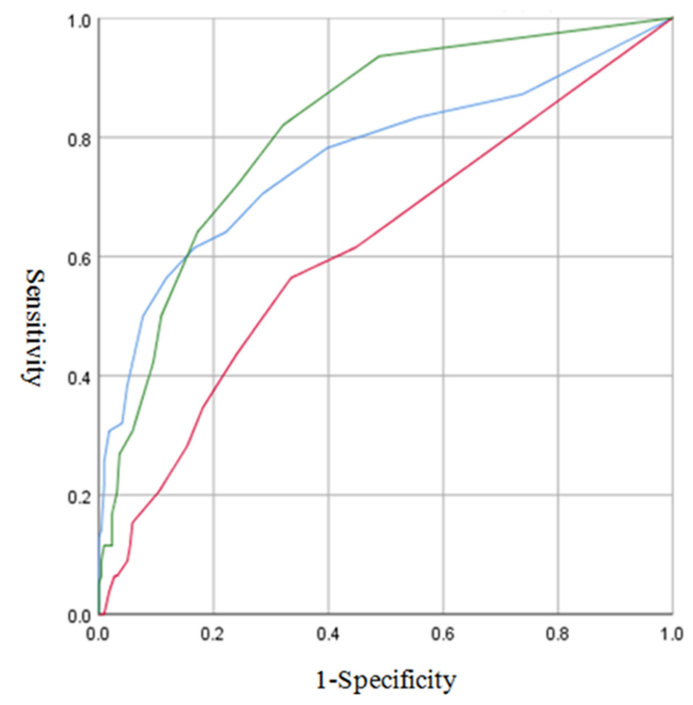

\footnotetext{
Source of the Curves

- ISI of baseline

- GAD-7 of baseline

PHQ-9 3 days after surgery
}

Figure 2 ROC curve of the risk factors.

Abbreviations: ROC, receiver operator characteristic; ISI, Insomnia Severity Index, GAD-7, Generalized Anxiety Disorder-7; PHQ-9, Patient Health Questionnaire-9. 
significant difference in the relationship between cognition and insomnia. So, it would seem more research is needed to confirm a potential link. We have also found no strong evidence that insomnia is related to pain. Our study was consistent with that of Chen et al, ${ }^{25}$ which indicated no correlation between sleep quality and pain. Previous research has suggested a relationship between the experience of pain and sleep disruptions among patients undergoing surgery, in that more severe pain is associated with more frequent awakenings throughout the night. ${ }^{26}$ The possible reason for the different conclusions may be that we only observed pain and insomnia within three days of surgery, and the long-term condition was not investigated.

The findings in the present study are in broad agreement with previous studies, which have reported that poor sleep quality is associated with poorer health status, disease severity, impaired daily activities, and reduced survival. ${ }^{27,28}$ This study acts as a reminder of the need to enquire about sleep quality in patients having an operation and to consider the investigation of potentially treatable causes, including measures to improve factors that adversely affect sleep. Our study also underlines the need for specific recommendations for the management of patients' sleep, which could greatly benefit their health. Preoperative counseling, early postoperative sleep modifications, and, possibly, preemptive use of medications may improve transient sleep disturbance among surgical patients. There is a need to manage sleep disturbance routinely in clinical practice and to screen for sleeping problems. Further studies are warranted concerning what factors contribute to sleep disturbance and how they can be best treated. This study makes an important contribution to the literature in that it adds to the existing evidence linking insomnia to depression and anxiety. Insomnia is often forgotten, but since a high percentage of patients are affected by it, it may well be of considerable clinical significance.

There is a need for the treatment and prevention of anxiety-related disorders. Doctors must manage patients' expectations whilst working with them to optimize sleep quality. Several "biopsychosocial" mechanisms hold promise as possible treatment targets for sleep interventions to reduce both insomnia and its symptoms. ${ }^{29}$ Cognitive behavior therapy has been shown to be effective at reducing insomnia and depression severity for older adults, and it has been demonstrated that mental health services can improve recovery outcomes for older adults with depression. ${ }^{30}$ It is important to design interventions aimed at decreasing the effects of insomnia in surgical patients to improve their health and quality of life. The ever-increasing rate of sleep disorders means identifying modifiable risk factors is of the utmost importance. Moreover, as insomnia exists in so many patients during the perioperative stage, correctly diagnosing and treating the sleep problems of individual patients is essential. The timely and effective management of such problems is beneficial from a clinical standpoint, as they can otherwise exacerbate certain associated symptoms, such as poor concentration or irritability.

\section{Limitations}

We recognize that there are several limitations to our study. First, it was based on data from a retrospective study, and certain clinical data related to sleep disorders, such as sleep duration time, were not collected. Moreover, we only recorded clinical information within three days of surgery, and the long-term postoperative complications were not observed. This study only investigated the relationship between short-term postoperative insomnia and mood. However, cohort projects with several long-term follow-ups could play a better predictive role. In the present study, anxiety and depression may only contribute to a temporary explanation of insomnia. Finally, we did not record the sleep conditions of other patients in the same ward, which may have had a significant influence on the participants.

\section{Conclusion}

Insomnia is common in patients and can worsen after surgery. The present study suggests that depression and anxiety are risk factors for insomnia after surgery. Our findings suggest that there is a link between sleep impairment in patients and a worsening health status. This finding is of considerable clinical relevance. Since patients undergoing an operation often report insomnia, there is a need for further research into strategies for managing depression and anxiety so that such patients may sleep better and enjoy significant health benefits.

\section{Data Sharing Statement}

The authors intend to share data on individuals identified as participants. We declared that materials described in the manuscript, including all relevant raw data, will be freely available to any scientist wishing to use them for non-commercial purposes, without breaching participant confidentiality. No other study documents will be made 
available. The data can be downloaded free from Dove's website. Data availability takes effect from publication in the SCI database and can be referenced all the time the data is published online in the SCI database.

\section{Ethics Approval and Consent to Participate}

The study was conducted in accordance with the Declaration of Helsinki (as was revised in 2013). The study was approved by the Ethics Committee of Beijing Chao-Yang Hospital (NO.2019-ke-273). Informed consent was obtained from all individual participants included in the study.

\section{Acknowledgments}

We are particularly grateful to all the people who have given us help with our article.

\section{Author Contributions}

All authors made substantial contributions to conception and design, acquisition of data, or analysis and interpretation of data; took part in drafting the article or revising it critically for important intellectual content; agreed to submit to the current journal; gave final approval of the version to be published; and agree to be accountable for all aspects of the work.

\section{Funding}

This study was funded by: Beijing Hospitals Authority Youth Programme (No.QML20190307); The Youth Backbone of Beijing Outstanding Talent Training Project (No. 2018000021469G215); Beijing Natural Science Foundation (No.7194270). The funding body had no role in the design of the study and collection, analysis, and interpretation of data and in writing the manuscript.

\section{Disclosure}

The authors declare that they have no competing interests.

\section{References}

1. Ierodiakonou D, Bouloukaki I, Kampouraki M, et al. Subjective sleep quality is associated with disease status in COPD patients. The cross-sectional Greek UNLOCK study. Sleep Breath. 2020;24:1599-1605. doi:10.1007/s11325-020-02039-8

2. Ozdemir PG, Boysan M, Selvi Y, Yildirim A, Yilmaz E. Psychometric properties of the Turkish version of the sleep hygiene index in clinical and non-clinical samples. Compr Psychiatry. 2015;59:135-140. doi:10.1016/j.comppsych.2015.02.001
3. Baglioni C, Riemann D. Is chronic insomnia a precursor to major depression? Epidemiological and biological findings. Curr Psychiatry Rep. 2012;14(5):511-518. doi:10.1007/s11920-012-0308-5

4. Manning BT, Kearns SM, Bohl DD, Edmiston T, Sporer SM, Levine BR. Prospective assessment of sleep quality before and after primary total joint replacement. Orthopedics. 2017;40(4):e636-e640. doi:10.3928/01477447-20170411-01

5. Halle IH, Westgaard TK, Wahba A, Oksholm T, Rustøen T, Gjeilo KH. Trajectory of sleep disturbances in patients undergoing lung cancer surgery: a prospective study. Interact Cardiovasc Thorac Surg. 2017;25(2):285-291. doi:10.1093/icvts/ivx076

6. Induru RR, Walsh D. Cancer-related insomnia. Am J Hosp Palliat Care. 2014;31(7):777-785. doi:10.1177/1049909113508302

7. Gagnon C, Bélanger L, Ivers H, Morin CM. Validation of the Insomnia severity index in primary care. J Am Board Fam Med. 2013;26(6):701-710. doi:10.3122/jabfm.2013.06.130064

8. Spitzer RL, Kroenke K, Williams JB. Validation and utility of a self-report version of PRIME-MD: the PHQ primary care study. primary care evaluation of mental disorders. Patient health questionnaire. JAMA. 1999;282(18):1737-1744. doi:10.1001/ jama.282.18.1737

9. Arrieta J, Aguerrebere M, Raviola G, et al. Validity and utility of the patient health questionnaire (PHQ)-2 and PHQ-9 for screening and diagnosis of depression in Rural Chiapas, Mexico: A cross-sectional study. J Clin Psychol. 2017;73(9):1076-1090. doi:10.1002/jclp.22390

10. Dear BF, Titov N, Sunderland M, et al. Psychometric comparison of the generalized anxiety disorder scale-7 and the penn state worry questionnaire for measuring response during treatment of generalised anxiety disorder. Cogn Behav Ther. 2011;40(3):216-227. doi:10.1080/16506073.2011.582138

11. Esser P, Hartung TJ, Friedrich M, et al. The Generalized Anxiety Disorder Screener (GAD-7) and the anxiety module of the Hospital and Depression Scale (HADS-A) as screening tools for generalized anxiety disorder among cancer patients. Psychooncology. 2018;27 (6):1509-1516. doi:10.1002/pon.4681

12. Olson RA, Iverson GL, Carolan H, Parkinson M, Brooks BL, McKenzie M. Prospective comparison of two cognitive screening tests: diagnostic accuracy and correlation with community integration and quality of life. J Neurooncol. 2011;105(2):337-344. doi:10.1007/ s11060-011-0595-4

13. Lai J, Ma S, Wang Y, et al. Factors associated with mental health outcomes among health care workers exposed to Coronavirus disease. JAMA Netw Open. 2019;3(3):e203976. doi:10.1001/ jamanetworkopen.2020.3976

14. Lin VY, Chung J, Callahan BL, et al. Development of cognitive screening test for the severely hearing impaired: hearing-impaired MoCA. Laryngoscope. 2017;127(Suppl 1):S4-S11. doi:10.1002/ lary. 26590

15. Trzepacz PT, Hochstetler H, Wang S, et al. Relationship between the montreal cognitive assessment and mini-mental State Examination for assessment of mild cognitive impairment in older adults. $B M C$ Geriatr. 2015;15:107. doi:10.1186/s12877-015-0103-3

16. Roth T, Coulouvrat C, Hajak G, et al. Prevalence and perceived health associated with insomnia based on DSM-IV-TR; international statistical classification of diseases and related health problems, tenth revision; and research diagnostic criteria/international classification of sleep disorders, Second Edition criteria: results from the America Insomnia Survey. Biol Psychiatry. 2011;69(6):592-600. doi:10.1016/ j.biopsych.2010.10.023

17. Le Grande MR, Jackson AC, Murphy BM, Thomason N. Relationship between sleep disturbance, depression and anxiety in the 12 months following a cardiac event. Psychol Health Med. 2016;21(1):52-59. doi:10.1080/13548506.2015.1040032 
18. Zhai L, Zhang H, Zhang D. Sleep duration and depression among adults: a meta-analysis of prospective studies. Depress Anxiety. 2015;32(9):664-670. doi:10.1002/da.22386

19. Yu J, Rawtaer I, Fam J, et al. Sleep correlates of depression and anxiety in an elderly Asian population. Psychogeriatrics. 2016;16 (3):191-195. doi:10.1111/psyg.12138

20. Yang Y, Zhu DM, Zhang C, et al. Brain structural and functional alterations specific to low sleep efficiency in major depressive disorder. Front Neurosci. 2020;14:50. doi:10.3389/fnins.2020.00050

21. Mansano-Schlosser TC, Ceolim MF, Valerio TD. Poor sleep quality, depression and hope before breast cancer surgery. Appl Nurs Res. 2017;34:7-11. doi:10.1016/j.apnr.2016.11.010

22. Ju YE, McLeland JS, Toedebusch CD, et al. Sleep quality and preclinical Alzheimer disease. JAMA Neurol. 2013;70(5):587-593. doi:10.1001/jamaneurol.2013.2334

23. Wang C, Holtzman DM. Bidirectional relationship between sleep and Alzheimer's disease: role of amyloid, tau, and other factors. Neuropsychopharmacology. 2020;45(1):104-120. doi:10.1038/ s41386-019-0478-5

24. Walker MP. The role of slow wave sleep in memory processing. J Clin Sleep Med. 2009;5(2 Suppl):S20-S26. doi:10.5664/ jcsm.5.2S.S20

25. Chen AF, Orozco FR, Austin LS, Post ZD, Deirmengian CA, Ong AC. Prospective evaluation of sleep disturbances after total knee arthroplasty. J Arthroplasty. 2016;31(1):330-332. doi:10.1016/ j.arth.2015.07.044
26. Herrero-Sánchez MD, García-Iñigo Mdel C, Nuño-Beato-Redondo BS, Fernández-de-las-peñas C, Alburquerque-Sendín F. Association between ongoing pain intensity, health-related quality of life, disability and quality of sleep in elderly people with total knee arthroplasty. Cien Saude Colet. 2014;19(6):1881-1888. doi:10.1590/141381232014196.04632013

27. Grandner MA, Malhotra A. Connecting insomnia, sleep apnoea and depression. Respirology. 2017;22(7):1249-1250. doi:10.1111/ resp. 13090

28. Castro-Marrero J, Zaragozá MC, González-Garcia S, et al. Poor self-reported sleep quality and health-related quality of life in patients with chronic fatigue syndrome/myalgic encephalomyelitis. J Sleep Res. 2018;27(6):e12703. doi:10.1111/jsr.12703

29. Blake MJ, Trinder JA, Allen NB. Mechanisms underlying the association between insomnia, anxiety, and depression in adolescence: implications for behavioral sleep interventions. Clin Psychol Rev. 2018;63:25-40. doi:10.1016/j.cpr.2018.05.006

30. Sadler P, McLaren S, Klein B, Harvey J, Jenkins M. Cognitive behavior therapy for older adults with insomnia and depression: a randomized controlled trial in community mental health services. Sleep. 2018;41(8). doi:10.1093/sleep/zsy104

\section{Publish your work in this journal}

Neuropsychiatric Disease and Treatment is an international, peerreviewed journal of clinical therapeutics and pharmacology focusing on concise rapid reporting of clinical or pre-clinical studies on a range of neuropsychiatric and neurological disorders. This journal is indexed on PubMed Central, the 'PsycINFO' database and CAS, and is the official journal of The International Neuropsychiatric Association (INA). The manuscript management system is completely online and includes a very quick and fair peer-review system, which is all easy to use. Visit http://www.dovepress.com/testimonials.php to read real quotes from published authors. 